\title{
Ecología, tecnología e innovación para la sustentabilidad: retos y perspectivas en México
}

Ecology, technology and innovation towards sustainability: challenges and perspectives in Mexico

Mayra E. Gavito $^{\mathrm{a}, *}$, Hans van der Wal ${ }^{\mathrm{b}}$, E. Miriam Aldasoro ${ }^{\mathrm{b}}$, Bárbara Ayala-Orozco ${ }^{\mathrm{a}}$, Aída Atenea Bullén ${ }^{a}$, Manuel Cach-Pérez ${ }^{b}$, Alejandro Casas-Fernández ${ }^{a}$, Alfredo Fuentes ${ }^{a}$, Carlos González-Esquivel ${ }^{\mathrm{a}}$, Pablo Jaramillo-López ${ }^{\mathrm{a}}$, Pablo Martínez ${ }^{\mathrm{b}}$, Omar Masera-Cerruti ${ }^{\mathrm{a}}$, Fermín Pascual $^{\mathrm{a}}$, Diego R. Pérez-Salicrup ${ }^{\mathrm{a}}$, Ramiro Robles ${ }^{\mathrm{b}}$, Ilse Ruiz-Mercado ${ }^{\mathrm{a}}$ y Gilberto Villanueva ${ }^{b}$

\footnotetext{
a Instituto de Investigaciones en Ecosistemas y Sustentabilidad, Universidad Nacional Autónoma de México, Campus Morelia, Antigua carretera a Pátzcuaro 8701, Colonia Ex-Hacienda San José de la Huerta, 58090 Morelia, Michoacán, México

${ }^{\mathrm{b}}$ El Colegio de la Frontera Sur, Unidad Villahermosa, Carretera a Reforma Km 15.5 s/n, Ra. Guineo Segunda Sección, Centro, 86280 Villahermosa, Tabasco, México
}

Recibido el 28 de noviembre de 2016; aceptado el 12 de septiembre de 2017

Disponible en Internet el 10 de noviembre de 2017

\section{Resumen}

En México y en el mundo, la incorporación de la comunidad científica (entre ellos los ecólogos) a la generación de conocimientos que coadyuven a solucionar los graves problemas ambientales, y avanzar hacia la sustentabilidad, requiere de una visión diferente en la investigación. Más aún, se requiere de herramientas novedosas para acoplar el trabajo de la comunidad científica con el resto de la sociedad. Los ecólogos tienen el reto de volverse innovadores y creadores de nuevos modelos, procesos de colaboración, métodos y herramientas de investigación, básica y aplicada, en temas como la agricultura, la forestería, la ganadería y el uso de los recursos naturales. Para enfrentar este reto, los ecólogos en México necesitan amalgamar su quehacer con el de otros actores académicos y no académicos para impulsar en conjunto procesos de mejoramiento ambiental con beneficios sociales. En esta contribución se revisan retos y perspectivas que se tienen en México en el campo de innovación ecotecnológica. Se propone fomentar el trabajo de investigación en laboratorios sin muros, en los cuales grupos flexibles de actores diseñen, prueben y evalúen innovaciones tecnológicas que respondan a problemáticas ambientales y socioecológicas locales y regionales específicas.

(C) 2017 Universidad Nacional Autónoma de México, Instituto de Biología. Este es un artículo Open Access bajo la licencia CC BY-NC-ND (http://creativecommons.org/licenses/by-nc-nd/4.0/).

Palabras clave: Ecotecnología; Educación; Capacitación; Manejo; Recursos naturales

\section{Abstract}

In Mexico and in the world, the incorporation of the scientific community (among them ecologists) to the generation of knowledge that contributes to solving the serious environmental problems, and advancing towards the sustainability, requires a different research vision. Moreover, innovative tools are needed to couple the work of the scientific community with the rest of the society. Ecologists are challenged to become innovators and

\footnotetext{
* Autor para correspondencia.

Correo electrónico: mgavito@ cieco.unam.mx (M.E. Gavito).

La revisión por pares es responsabilidad de la Universidad Nacional Autónoma de México.
} 
creators of new models, collaborative processes, methods and research tools, basic and applied, in topics such as agriculture, forestry, livestock and the use of natural resources. To meet this challenge, ecologists in Mexico need to amalgamate their work with other academic and non-academic actors to jointly promote environmental improvement processes with social benefits. This contribution examines challenges and perspectives of ecotechnological innovation in Mexico. We suggest to foster the research work in this field in laboratories without walls, in which flexible groups of actors design, test, and evaluate ecotechnological innovations that respond to specific local and regional socio-ecological and environmental problems.

(C) 2017 Universidad Nacional Autónoma de México, Instituto de Biología. This is an open access article under the CC BY-NC-ND license (http://creativecommons.org/licenses/by-nc-nd/4.0/).

Keywords: Ecotechnology; Education; Training; Management; Natural resources

\section{Introducción}

La extinción de especies en el Antropoceno (Crutzen y Stoermer, 2000), el cambio climático global y los daños a los ecosistemas naturales ocasionados por las actividades humanas, son parte de una crisis ambiental y socioecológica que se manifiesta a escalas locales, regionales y global (Milennium Ecosystem Assessment, 2005). Esta crisis pone en entredicho la convivencia social y las bases de la propia existencia de la humanidad. Los 17 Objetivos de Desarrollo Sostenible de la Agenda 2030 para el Desarrollo Sostenible (ONU, 2015) marcan un esfuerzo sin precedentes para atender esta crisis. Sin embargo, estos objetivos son también indicativos de un marco general de pobreza, hambre, inseguridad alimentaria, desnutrición, prácticas agrícolas insostenibles, inequidad (económica, social y de género), despilfarro de recursos naturales, consumo energético desmesurado y de violencia e inseguridad (ONU, 2015).

La atención a los problemas del uso y manejo insostenible de recursos naturales es de máxima relevancia en el contexto anterior. Uno de los grandes retos para transitar hacia la sustentabilidad es lograr que la sociedad, en su conjunto, participe en la construcción de soluciones a estos problemas. Para ello, se requiere de nuevos modelos de desarrollo basados en el uso sustentable de los ecosistemas y sus recursos renovables, que minimice la degradación ambiental. La comunidad científica, entre ellos los ecólogos, puede contribuir a generar tales modelos (Carpenter y Gunderson, 2001). Sin embargo, esta comunidad no puede realizar por sí sola esta tarea, ya que incluso los más brillantes desarrollos científicos y tecnológicos no están logrando vencer el reto de frenar el deterioro ambiental, cuya magnitud y complejidad no tienen paralelo en la historia de la humanidad. Para ello, se requiere integrar una plataforma de actores académicos y no académicos capaces de construir a un mismo tiempo la concientización social de la problemática ambiental, gestar el interés común para solucionarla y aportar respuestas de acción que continuamente se evalúen e innoven.

La crisis ambiental y socioecológica se manifiesta en cada país con particularidades propias debido a la combinación sui generis de factores naturales, sociales, económicos, culturales y políticos. En el caso de México, la confluencia de 2 factores es especialmente relevante en la búsqueda de respuestas y soluciones: la megadiversidad biológica (Conabio, 1998) y la megadiversidad cultural y social existentes en el país. Estas 2 particularidades encierran una gama enorme de conocimiento, creatividad y trayectoria en la gestión de alternativas de sobrevivencia en múltiples contextos de crisis repentinos (terremotos, inundaciones, epidemias, crisis económicas, erupciones volcánicas), de larga duración (e.g., cambio climático global) y estructurales (como la inequidad y la pobreza). En este sentido, México puede considerarse como un megalaboratorio con grandes variantes socioecológicas, en mundos urbanos tan divergentes como las ciudades de Tijuana, San Cristóbal de las Casas, Cancún y la Ciudad de México, en entornos rurales de producción agrícola industrial (e.g., el Bajío o Sinaloa) y en comunidades rurales que desarrollan actividades de agricultura y ganadería familiar (como aquellas presentes en los estados de Oaxaca, Michoacán, Guerrero y Chiapas). Son precisamente estos laboratorios vivientes los que pueden ser catalizados en su actividad innovadora y de adaptación a través de alianzas entre profesionales y expertos en temas ambientales relevantes, que incluyen a los académicos de las ciencias naturales y sociales.

Globalmente se establecen cada vez más los llamados «laboratorios sin muros», que si bien pueden anidarse en las universidades y centros de investigación, operan en composiciones y espacios flexibles, de acuerdo con las problemáticas que atienden, adaptando su composición a las necesidades del caso (Espinoza-Tenorio, Mesa-Jurado, Ortega-Argueta y Hernández-Chávez, 2017). Estos laboratorios pueden reunir desde capacidades técnicas (como el de modeladores, informáticos, mecánicos, plomeros y carpinteros) hasta capacidades científicas con investigadores en diferentes áreas de interés. Estos laboratorios permiten la confluencia de experiencias profesionales diversas para atender una problemática de la sociedad (Espinoza-Tenorio et al., 2017).

El modelo de laboratorio sin muros es particularmente adecuado para la atención de temas que, por su complejidad, requieren de grupos de trabajo transdisciplinarios, aquel en el que participan académicos y actores no académicos. En la actualidad, con frecuencia tales grupos solo se dan de manera incidental como ocurre, por ejemplo, a través de proyectos de financiamiento temporal, cuyas experiencias no son sistematizadas y que, por lo mismo, no conducen a los aprendizajes que se requieren para la solución del problema. Si bien en México existen múltiples experiencias de este tipo (Ortiz, Masera y Fuentes, 2014), estas presentan fuertes limitaciones de falta de registro de datos, diferencias de procedimientos/metodologías y posiciones personales que impiden el desarrollo, el aprendizaje y la transmisión del conocimiento generado. Para la construcción de conocimientos y el desarrollo de probables soluciones a los grandes problemas ambientales se requiere de la participación 
de la comunidad científica proveniente de diversas disciplinas. En este sentido, la tecnología se ha convertido en la interfaz y el articulador o mediador natural entre la sociedad y el ambiente (Smith y Stirling, 2010; Redman y Miller, 2015).

Dado que el campo de trabajo de innovación ecotecnológica es reciente en México, más que desarrollar una revisión de la literatura y el estado del arte, en este artículo pretendemos, desde nuestro punto de vista, abordar algunos de los retos y perspectivas más importantes que vislumbramos en este campo para el país. Expondremos el porqué la innovación ecotecnológica es un medio que puede lograr la fusión de la ciencia y la sociedad en la búsqueda de la sustentabilidad, usando como sistemas de análisis a aquellos que tienen que ver con la producción agrícola, pecuaria y forestal. Finalmente, enfatizamos que la comunidad de ecólogos debería ser más innovadora en la búsqueda de esa fusión.

\section{Tecnología e innovación: el enfoque ecotecnológico}

Entendemos por tecnología, de manera general, la aplicación del conocimiento para generar nuevos métodos, procesos, servicios y dispositivos. Innovación tecnológica, por otro lado, es la transformación de una idea en un producto, equipo o proceso operativo, incluyendo nuevas formas de organización social (García y Calantone, 2002). Al estudiar la innovación tecnológica, se debe incluir el análisis de los procesos de generación/adopción/implementación/monitoreo y evaluación de las tecnologías.

El modelo convencional de desarrollo e innovación tecnológica considera que esta es inherentemente beneficiosa y resultado de un proceso natural que va de la ciencia básica a la ciencia aplicada y de esta a la tecnología (Vega, 2012). La tecnología llega a la sociedad mediante su comercialización por empresas que desarrollan patentes para proteger su propiedad intelectual. Este modelo lineal de innovación tecnológica ha sido fuertemente cuestionado ya que, por un lado, muchas tecnologías e innovaciones son altamente contaminantes, generan riesgos sanitarios, erosionan la diversidad biológica y cultural, agotan los recursos naturales y, en consecuencia, no conservan los recursos, no mejoran la calidad de vida, ni son sustentables (Cannatelli, Masi y Molteni, 2012; Olsson y Galaz, 2012). Por otro lado, muchas no son accesibles ni están adaptadas a las condiciones socioambientales de los países en desarrollo, particularmente en los sectores rurales. Se enfocan a atender una demanda o un mercado y no a las necesidades sociales (Cannatelli et al., 2012). Por esta razón, históricamente han surgido diferentes movimientos que han propuesto modelos alternativos de generación y aplicación de la tecnología. Uno de ellos es la ecotecnología, definida como los «dispositivos, métodos y procesos que propician una relación armónica con el ambiente y buscan brindar beneficios sociales y económicos tangibles a sus usuarios, con referencia a un contexto socioecológico específico» (Ortiz et al., 2014; Straškava, 1993). Otros ejemplos son la «Tecnología Apropiada», que buscaba ser de bajo costo y consumo energético, así como de uso local y colectivo (Thomas, 2012), y la «Tecnología Alternativa», que pretende la construcción de modelos sociales con comunidades descentralizadas que buscan ser autosuficientes con el uso de recursos renovables y pocos insumos (Fressoli, Smith, Thomas y Bortz, 2015). En este contexto, la innovación ecotecnológica sería la generación conjunta, también denominada «innovación inclusiva», de nuevos dispositivos, métodos y procesos. En este nuevo marco, los usuarios no son simplemente receptores de las tecnologías que desarrollan las empresas y centros de investigación, sino actores importantes en el proceso de desarrollo, en el cual aportan sus conocimientos y se atienden sus necesidades y prioridades (Fressoli, Dias y Thomas, 2014; Gupta et al., 2003). Es decir, la innovación ecotecnológica hace énfasis en ligar y armonizar la generación continua de conocimiento -que puede ser científico en el sentido estricto, o provenir del «diálogo de saberes» con las comunidades y actores socialespara convertirlo en nuevos productos/procesos/métodos.

La innovación ecotecnológica busca desarrollar tecnología que responda a necesidades concretas de diferentes actores sociales, como campesinos, empresas, comunidades rurales, organizaciones sociales. Su fin es el de mejorar la calidad ambiental (i.e., hacer más eficiente, conservar, restaurar, o remediar los recursos base y sus ecosistemas asociados), brindando impactos positivos a la sociedad (Fressoli et al., 2015). En particular se hace énfasis en desarrollar alternativas para los sectores sociales más vulnerables, pues han sido históricamente los que generalmente han quedado fuera de los procesos de innovación tecnológica, pero no significa que esto implique un marco excluyente para proyectos dirigidos a otros sectores.

Uno de los temas y retos más importantes para la innovación ecotecnológica es el uso sustentable de los recursos naturales. La innovación que se requiere para ello no se limita a introducir una novedad (en inglés «add on») sino que abarca desde innovar en la forma de ser del «agente» de innovación, de las instituciones y de las formas de trabajo, hasta las relaciones con los actores locales. Si en una visión tradicional el extensionista llevaba una técnica al campesino y la transmitía, mal o bien, ahora se trata de generar y aplicar conocimientos en procesos entre distintos actores, de facilitar estos procesos e incidir colectivamente en los marcos institucionales y las políticas. Se trata de una variante mejorada de la investigación-acción, con componentes y actores adicionales, de procesos de aprendizaje sistémico en temas complejos, que requieren de la interdisciplina, de la gestión y de la combinación iterativa de respuestas cada vez más certeras, que consideren el contexto socioecológico local y regional particulares.

Actualmente la tecnología ha adquirido una dimensión tan importante y globalizadora que se habla ya de sistemas sociotecnoecológicos (SETS por sus siglas en inglés), o sociotecnoecosistemas (Redman y Miller, 2015), para hacer más evidente su rol como mediador entre los seres humanos y el ambiente. Dicho de otra manera, no se pueden entender cabalmente los impactos de la actividad humana sobre el ambiente, ni contribuir a solucionar las crisis ambientales, sin incorporar a la tecnología. La innovación ecotecnológica dirigida hacia la sustentabilidad se logrará solo a través de la interacción con la sociedad, del seguimiento de todo el proceso desde su concepción hasta la apropiación, y de la retroalimentación entre los actores. El cambio requiere de enfoques integrales y 
transdisciplinarios que amalgamen a la ciencia con las necesidades de la sociedad, liguen la investigación básica y aplicada con la innovación, el desarrollo y la validación de tecnología, y verifiquen su posterior difusión y adopción por los actores sociales relevantes.

La innovación ecotecnológica puede aplicarse para desarrollar productos tan diversos como software para asistir a los actores gubernamentales en la planeación y en la toma de decisiones, técnicas para la remediación de suelos, sistemas eficientes de captación de agua o riego, prácticas agroecológicas en sistemas productivos, metodologías de evaluación de impactos ambientales, dispositivos eficientes para el uso de energía, entre muchos otros. No debe confundirse con la innovación biotecnológica, que en la práctica se refiere a áreas muy específicas (principalmente biomedicina, insumos agrícolas y pecuarios) y cuenta con numerosos ejemplos de innovaciones que se comercializan con la propaganda de ser alternativas sustentables, pero que no consideran los riesgos sanitarios, ni la pérdida de diversidad biológica o cultural, ni la degradación ambiental y mucho menos la sustentabilidad. Aunque algunas innovaciones partan de la intención de mejorar el bienestar de las personas, el modelo de desarrollo de estas innovaciones, así como la preponderancia de mercados económicos y la rentabilidad en sus criterios de éxito, con frecuencia no conducen a la sustentabilidad y a la apropiación social (Cannatelli et al., 2012). A diferencia de la innovación biotecnológica en biomedicina y agricultura, en la que se ha documentado un crecimiento importante en México y en el mundo, y en la que ya participan muchas instituciones públicas y privadas en los llamados sistemas de innovación, la biotecnología ambiental (que es el área que más se asocia con la ecotecnología) tiene un desarrollo incipiente y aún concentrado en manejo de agua, energía y residuos (Amaro-Rosales y Morales-Sánchez, 2010).

Gran parte de la ecotecnología que se está desarrollando en el país es doméstica, pero no por ello está desconectada de los problemas ambientales y del uso irracional de los recursos naturales (Ortiz et al., 2014). Hay mucha tarea para los ecólogos en la innovación ecotecnológica doméstica, la cual requiere tanto rigor en la modelación espacial y temporal del uso de los recursos (por ejemplo, en el aprovechamiento de las poblaciones y comunidades bióticas), o en la evaluación de los impactos humanos o desastres naturales sobre los ecosistemas, como lo requiere una innovación agrícola o una innovación para el manejo de cuencas. La innovación en el uso y conservación de los recursos naturales es un área crítica dentro de la ecotecnología pero aún es incipiente en México. Esta ha sido más impulsada en temas de energía tanto por la crisis energética como por la presión internacional para frenar el calentamiento global (Ortiz et al., 2014), que en otros recursos naturales relacionados con agua, suelo y biodiversidad. Aún dentro del contexto de la ecotecnología, muchas innovaciones en energía que buscan ser «verdes» y renovables no se enmarcan en políticas de verdadero desarrollo sustentable, donde entra en juego la preservación del bienestar social, del ambiente y de la naturaleza. En el caso del agua, siendo México un país con extensas zonas áridas, semiáridas o con regímenes de precipitación pluvial muy estacionales (Semarnat, 2014a), es prioritario desarrollar un manejo innovador para la captación, el almacenamiento, la eficiencia en el uso, el saneamiento, la purificación y el reciclaje de este recurso vital. Mínimamente se debe trabajar en reducir el Índice de Pobreza de Agua para la sociedad (Water Poverty Index, WPI, Cho, Ogwang y Opio, 2010), pero idealmente, igual que en energía, suelo y biodiversidad, debería ser una política permanente e integral hacia el desarrollo sustentable.

Más del $90 \%$ de los alimentos depende de actividades agrícolas en las que la calidad del suelo es crítica. Los suelos saludables se tornan más escasos conforme continuamos sobreexplotando los sistemas agrícolas (Pimentel et al., 1995) y cambiando el uso de suelo de ecosistemas naturales a productivos (Lambin et al., 2001). Si bien es cierto que esto sucede en todo el mundo, México pierde ecosistemas naturales saludables a una tasa alarmante (Challenger y Dirzo, 2009; Zika y Erb, 2009). La degradación del suelo y la desertificación en México son problemas serios que deben reducirse y revertirse con el fin de que la sociedad siga contando con los servicios ecosistémicos de suelos sanos y productivos (Huber-Sannwald, Maestre, Herrick y Reynolds, 2006). Se han desarrollado varias alternativas de manejo e intervenciones experimentales con diferentes niveles de éxito dependiendo del problema, la región geográfica, el tipo de ecosistema, el tipo de suelo y el grado de degradación (Cotler et al., 2007). Sin embargo, estos seguirán siendo esfuerzos desarticulados y de bajo impacto en tanto no formen parte de una estrategia nacional real de manejo sustentable. Existe una gran necesidad de un mejor inventario de suelos, de una colección sistemática de datos y su incorporación en bases computacionales bien organizadas que ayuden, por un lado, al trabajo de científicos y, por el otro, a los diseñadores de políticas públicas e instituciones gubernamentales en la toma de decisiones. El progreso que se ha logrado en el inventario de la biodiversidad y en la inclusión de la conservación de la biodiversidad en la agenda política con el trabajo de la Conabio muestra que las instituciones pueden promover grandes avances en temas que antes eran ignorados (Dirzo, González y March, 2009).

Además del manejo de los recursos naturales, otra área urgente de innovación ecotecnológica e involucramiento de la comunidad científica es el manejo sustentable de las tierras en actividades productivas. A escalas espaciales grandes (paisaje, cuenca, región) se hace evidente el impacto ambiental negativo que tienen las actividades productivas en los ecosistemas naturales, las cuales alteran en cascada a todos los componentes bióticos y abióticos, independientemente de si estos se encuentran bajo la acción directa del manejo o fuera de ella (Bunge, Cotler, Iura-González y Ruiz-Bedolla, 2014).

\section{Innovación para el manejo de sistemas productivos}

La innovación ecotecnológica en sistemas productivos agrícolas, ganaderos y forestales es radicalmente distinta a lo que se instrumentó en la llamada Revolución Verde, ya que en esta última se considera a los productores y sus familias solo como los receptores de las tecnologías que desarrollan las empresas y los centros de investigación. La Revolución Verde, iniciada en los años de los setentas del siglo pasado, aumentó en el corto plazo los rendimientos de los cultivos más importantes 
Tabla 1

Metas para el desarrollo sustentable en los sistemas productivos agrícolas, pecuarios y forestales y ejemplos de herramientas ecotecnológicas que pueden ayudar a lograr esas metas.

Metas para el desarrollo sustentable

Manejo eficiente del agua de lluvia y riego

Eficiencia de uso y reciclaje de energía renovable

Economía circular y estequiometría en nutrientes: balance de ingresos y egresos

Diversificación en tiempo y espacio de plantas, microorganismos y animales asociados con propósitos múltiples en el ecosistema productivo: fertilidad, control biológico, polinización, control de erosión, almacenamiento de agua, sombra, reciclaje

Variabilidad genética y redundancia funcional para fortalecer la resiliencia

Selección de sistemas de producción viables para las condiciones edáficas, climáticas y socioeconómicas

Manejo de disyuntivas y compensaciones para maximizar la provisión de servicios ecosistémicos

Manejo del paisaje para mantenimiento de recursos naturales y servicios ecosistémicos en diferentes escenarios de cambio de uso de suelo y cambio climático

Control de emisiones de gases de efecto invernadero y otros contaminantes ambientales
Ejemplos de herramientas ecotecnológicas para la sustentabilidad

Modelación, software de apoyo, diseño de sistemas de captación de agua y de riego eficiente, manejo de la cobertura vegetal, análisis de imágenes, diseño de indicadores de sequía, cursos de capacitación

Modelación espacial y temporal de los recursos energéticos, software de apoyo, diseño de sistemas de producción y reciclaje de energía en medios rurales, dispositivos de medición de eficiencia energética, alternativas para reducción de uso de energía, cursos de capacitación

Diseño de sistemas de producción basados en insumos locales, uso eficiente y reciclaje de nutrientes, análisis de ciclo de vida, software y aplicaciones para optimizar la fertilización, medir las entradas y salidas y generar balances estequiométricos, modelación espacial y temporal de disponibilidad y desbalances en nutrientes

Diseño de sistemas alternativos e integrales de producción con bases ecológicas, desarrollo de prácticas de cultivo e insumos locales para maximizar los beneficios sin deteriorar los recursos, sistemas de evaluación multicriterio, software y aplicaciones de asesoría para la intensificación sustentable, modelación de la capacidad de carga, manejo integrado de plagas y enfermedades, manejo integrado de cultivos, ganado y árboles, aprovechamiento de forrajes nativos y de subproductos agrícolas y agroindustriales, mapas para reforestación y regeneración natural de especies forestales

Modelación de poblaciones, estrategias de mantenimiento de la diversidad genética y la redundancia en funciones ecosistémicas

Software, bases de datos, modelación de climas y ajustes fisiológicos en plantas y animales, monitoreo de condiciones climáticas y eficiencia fisiológica para ajustes de fertilización y control de plagas

Software para evaluaciones de impacto ambiental y de sustentabilidad, modelación espacial y temporal de servicios, diseño de indicadores, análisis de riesgos y vulnerabilidades

Herramientas de percepción remota, análisis de imágenes, modelación espacial y temporal, mapas participativos, juegos de actores y toma de decisiones

Producción de fertilizantes con bajas emisiones, técnicas de manejo de excretas, compostas, biofertilizantes y bioplaguicidas, biodigestores adaptados a la producción ganadera de pequeña escala, software de asesoría en manejo de almacenes de carbono, fuegos prescritos o quemas de limpieza para la población mundial a través de la aplicación masiva de un paquete tecnológico simple y único (Evenson y Gollin, 2003). Sin embargo, se ha mostrado que es imposible lograr de esta manera ingresos dignos de la población rural campesina a la par de una relación sinérgica entre producción y conservación de los recursos naturales (Pingali, 2012). Por ejemplo, los modelos de producción convencional requieren de insumos externos (e.g., agroquímicos) que se van incrementando con el tiempo, ya que se basan en el reemplazo de los procesos ecológicos naturales por tecnologías de riego, fertilización y fitosanitarias artificiales; en la innovación ecotecnológica, por el contrario, se busca la diversificación de productos tomando como base a los procesos ecosistémicos naturales (Perfecto, Vandermeer y Wright, 2009). Esta última requiere de modelos productivos y de uso de los recursos naturales que reflejen las realidades socioculturales y ecológicas regionales y que, en un proceso hermenéutico de evaluación participativa, se ajuste la producción con el entorno ambiental. Los usuarios se vuelven actores importantes en este proceso, en el cual aportan sus conocimientos y se atienden sus necesidades y prioridades.

En el contexto actual de cambio global, en particular del calentamiento global, la población necesita ajustar el uso del suelo y la infraestructura a sus estrategias de manejo. Para ello, se requiere que la investigación básica y aplicada contribuya a un mayor conocimiento de cómo los agroecosistemas responden a la variación climática. Combinando este conocimiento científico con el conocimiento campesino, que sustenta a los sistemas tradicionales de producción, se pueden aportar innovaciones para el desarrollo de alternativas de sistemas alimentarios sustentables regionales. Innovación, adaptación, planeación, modelación son aspectos que requieren de la combinación de conocimiento local y científico sólido en ecología, sobre la relación entre variables climáticas y edáficas, las relaciones planta-suelo, interacciones bióticas, y ajustes fisiológicos de las plantas manejadas en el entorno del agroecosistema. No es adecuado ya construir estos conocimientos únicamente desde la perspectiva de los académicos y luego transferirla. La generación de innovación ecotecnológica dirigida a la producción agrícola sustentable depende de manera crítica del intercambio de conocimientos y la participación activa de los actores, incluyendo los productores, desde el inicio. El diseño de metas de producción sustentable requiere de métodos creativos y herramientas innovadoras, donde el conocimiento ecológico es central (tabla 1). 
Tabla 2

Ejemplo de innovación ecotecnológica en la producción de cacao en Tabasco.

El cacao (Theobroma cacao) es de rica tradición en las tierras bajas del trópico húmedo en Tabasco, México. A pesar de la acumulación de conocimiento sobre su cultivo a lo largo de los siglos, su integración en la cultura, e interés económico, actualmente la producción está inmersa en un síndrome de problemas, que incluye desorganización de productores, políticas equivocadas y enfermedades a consecuencia de un manejo deficiente. Sin embargo, hay oportunidades de innovación ecotecnológica en el cacao, debido a condiciones de mercado favorables y el arraigo cultural del cultivo. Tal innovación debería de componerse de una combinación de los siguientes elementos:

Talleres de análisis de: la producción y comercialización de cacao; evaluación de las oportunidades que brinda un mercado al alza; las exigencias de este mercado; la diferenciación del mercado de cacao; análisis del marco institucional actual; modelos de organización; técnicas de selección de semillas de calidad; cateo de los sabores del cacao; sistemas de control de calidad; sistemas de certificación

Diseño ecotecnológico: generación e instalación de tecnologías para la elaboración in situ de productos especiales a partir del cacao; generación de tecnologías de clasificación de semillas de cacao; selección de variedades nuevas de cacao; introducción de pataste (Theobroma bicolor) como alternativa al T. cacao; secado de cacao

Organización: formulación de nuevos modelos de asociación de empresas del cacao y organizaciones locales de productores e iniciarlos a escala piloto

Capacitación: contabilidad de cooperativas; cateo de cacao; certificación de la producción de distintos modelos de producción de cacao; uso del certificado de origen «cacao Grijalva»; la propagación sexual y vegetativa para obtener plántulas de cacao

Evaluación de plantaciones: evaluación ecofisiológica de cacaotales; evaluación de la productividad de clones de cacao; evaluación de la susceptibilidad a enfermedades de los clones de cacao; evaluación de opciones de diversificación del cacao; monitoreo de las condiciones ambientales en el cacaotal

\section{Innovación en sistemas agrícolas}

Agricultura, en el contexto actual de desertificación, calentamiento global, pérdida de biodiversidad y otras manifestaciones del cambio global, implica, además de producir alimentos, encontrar cómo se puede satisfacer esta demanda de manera combinada con: $i$ ) la conservación de (agro)ecosistemas con funciones ecológicas complementarias en mosaicos de usos diversos, ii) la aplicación de prácticas sustentables de manejo agrícola, ganadero y forestal, iii) un nivel de soberanía alimentaria y energética que garantice la supervivencia de la población durante eventos de crisis, $i v$ ) redes de comercialización y distribución de bienes y servicios múltiples de logística flexible, $v$ ) una organización social propensa a una cierta solidaridad (Altieri, Nicholls, Henao y Lana, 2015) y vi) un marco institucional y de gobernanza que facilite todos los aspectos anteriores (Ostrom, 2009).

La innovación ecotecnológica en agricultura y el uso de los recursos naturales implica el desarrollo de métodos y acciones para lograr los puntos indicados en el párrafo previo; es el aprendizaje social del sistema que resulta en su ajuste continuo. Es, en nuestra definición, lo que conduce a la sustentabilidad de un sistema socioecológico (Ostrom, 2007). Tal sistema es divisible en los subsistemas de los contenedores de los recursos (e.g., el agroecosistema, la selva, el humedal), del recurso aprovechado (la madera, el cultivo, los peces), de la gobernanza (las reglas, acuerdos, leyes e instituciones que rigen el acceso al recurso y su aprovechamiento), de los usuarios y de sus interacciones, que resultan en la condición final del sistema (en inglés el «outcome»). El sistema se encuentra inmerso en un sistema ecológico mayor, como también en un contexto social y político, que definen el marco de respuesta y adaptación del sistema. La innovación ecotecnológica abarca estos subsistemas e interacciones y propicia precisamente la sustentabilidad del socioecosistema agrícola (tabla 2).

Perfecto y Vandermeer (2015) analizando el cafetal, uno de los agroecosistemas más diversificados que se gesta a partir de la organización de productores y consumidores y que es compatible con la conservación de recursos naturales, consideran que (siguiendo a Levins y Lewontin, 1985) «... No es que el todo sea más que la suma de sus partes, sino que las partes adquieren nuevas propiedades. Sin embargo, al adquirir las partes nuevas propiedades, confieren nuevas propiedades al conjunto, que se reflejan en cambios en las partes, y así consecutivamente. Partes y conjuntos se desenvuelven en consecuencia de sus relaciones y la misma relación se desenvuelve». Tal es precisamente el caso de los sistemas productivos en la agricultura. En ellos el gran reto es el cambio de modelo de producción a un manejo integrado y diversificado que considere las metas establecidas en la tabla 2 , para las cuales es necesario desarrollar diferentes innovaciones ecotecnológicas. Lo mismo aplica en los sistemas pecuarios y forestales, si bien hay algunas particularidades en ellos como se remarca a continuación.

\section{Innovación ecotecnológica en sistemas pecuarios}

Los sistemas pecuarios merecen una mención especial en México porque la ganadería bovina, específicamente para la producción de carne, utiliza el $68 \%$ de la superficie nacional. No obstante son, a diferencia de los sistemas agrícolas y forestales, muy poco atendidos en los estudios ecológicos. La mayoría de los sistemas de producción ganadera se caracterizan por la utilización de grandes áreas de pastos con especies forrajeras (con frecuencia exóticas) de baja calidad nutritiva, con bajos niveles tecnológicos, con el empleo de dosis crecientes de agroquímicos $\mathrm{y}$, en general, con el uso excesivo de insumos externos (Tudela, 1992). La producción ganadera se ha expandido desde los años 1960 principalmente a costa de los bosques y otros ecosistemas naturales (Steinfeld y Chilonda, 2006). La conversión de vegetación a potreros y el manejo ganadero representan el $14.5 \%$ de las emisiones antropogénicas de gases de efecto invernadero (Opio et al., 2013). El 39\% de dichas emisiones corresponde al metano producido durante la fermentación ruminal. Otros impactos ambientales de la ganadería son la eutrofización de cuerpos de agua por lixiviación de nitrógeno, producto del uso excesivo de fertilizantes en forrajes cultivados y de alimentos concentrados; el deterioro del suelo por compactación y, paradójicamente, por empobrecimiento derivado del uso ineficiente de fertilizantes, el sobrepastoreo y el monocultivo (Mahecha, 2016). Dado que la demanda global de productos de origen animal seguirá incrementándose a medida que la población humana aumenta y que las economías emergentes crecen y aumenta el 
ingreso de las familias, es urgente desarrollar ecotecnologías que reduzcan los impactos ambientales negativos provocados por la ganadería convencional y extensiva.

Particularmente, se requiere generar ecotecnologías viables que permitan transitar de la ganadería extensiva a sistemas más intensivos, integrados y sostenibles (Semarnat, 2014a), acompañados de estrategias trans-sectoriales, con compromisos y responsabilidades internacionales y nacionales. Así, ecotecnologías silvopastoriles, agroforestales y agroecológicas, se han reconocido mundialmente como alternativas viables y al alcance de las poblaciones locales (Murgueitio e Ibrahim, 2001). Estas ecotecnologías utilizan especies arbóreas nativas que cumplen funciones múltiples como son producción de madera, leña, forraje, alimento y medicina, además de aportar servicios de sombra, fertilización de la tierra y almacenamiento de agua (Ibrahim, Villanueva, Casasola y Rojas, 2006). Los sistemas agroforestales pueden ordenarse en corredores biológicos. De este modo, al mismo tiempo que mejoran las prácticas de producción ganadera, mitigan los efectos del cambio climático, contribuyen a la conservación de la biodiversidad y reducen la deforestación o la degradación de los bosques, reduciendo emisiones de carbono y aumentando la captura de este gas en la parte forestal (Amézquita, Ibrahim, Llanderal, Buurman y Amézquita, 2004). En conjunto, estas ecotecnologías conforman estrategias versátiles para la mitigación y la adaptación al cambio climático global en México, mejorando la resiliencia de los sistemas productivos (Ferguson et al., 2013; Jiménez-Ferrer, Aguilar-Argüello y Soto-Pinto, 2008; Nahed-Toral et al., 2013; Villanueva, Martínez, Casanova, Ramírez y Montañez, 2015).

Para que estas ecotecnologías se conviertan en una innovación ampliamente aplicada, se requiere de experimentos locales y la evaluación de los resultados a través de talleres/trabajo participativo con los productores. También se requiere de acuerdos con las instituciones y profesionistas que actualmente propagan el uso de las tecnologías convencionales. Para ello, es necesario aplicar métodos didácticos freirianos (i.e., educación a través del diálogo), que reconozcan el conocimiento local.

\section{Innovación en el manejo de recursos forestales}

México se enfrenta al reto de manejar adecuadamente sus recursos forestales en un territorio privilegiado por una enorme biodiversidad y una extraordinaria diversidad cultural de pueblos que han practicado por siglos el aprovechamiento de múltiples recursos bióticos. Indudablemente, para transitar hacia un manejo integrado y sustentable de los ecosistemas naturales forestales, el primer desafío es dejar atrás la visión silvícola convencional (Pérez-Salicrup, 2005). Las innovaciones ecotecnológicas en este campo deben basarse en un sólido conocimiento ecológico sobre la estructura y dinámica de los ensambles de especies vegetales que constituyen los ecosistemas forestales, y su capacidad de recuperación ante los disturbios ocasionados por el aprovechamiento. El conocimiento generado a través de la investigación participativa resulta fundamental (MartínezTorres, Cantú-Férnandez, Ramírez-Ramírez y Pérez-Salicrup, 2015; Martínez-Torres, Castillo, Ramírez-Ramírez y Pérez-
Salicrup, 2016). La integración de la investigación ecológica con la experiencia de los manejadores de bosques ha demostrado ser más exitosa que las explotaciones monoespecíficas convencionales (Pinard, Guariguata, Putz y Pérez-Salicrup, 2006), o que aquellas que se experimentan en contextos tradicionales, para cubrir la demanda del mercado de ciertos productos específicos (Delgado-Lemus, Torres, Blancas y Casas, 2014; Rangel-Landa, Dávila y Casas, 2015; Torres, Blancas, León y Casas, 2015; Torres, Casas, Delgado-Lemus y Rangel-Landa, 2013; Torres, Casas, Vega, Martínez-Ramos y Delgado-Lemus, 2015).

Hoy en día, el aprovechamiento forestal hegemónico en México es el aprovechamiento maderable de unas cuantas especies de pino (Semarnat, 2014b). Las estadísticas de productos forestales no maderables indican que el producto de mayor aprovechamiento es la resina, que también se extrae de árboles de especies del género Pinus (Tapia-Tapia y Reyes-Chipla, 2008; Semarnat, 2014b). Sin embargo, el aprovechamiento forestal de bosques de pinos sigue basándose en sistemas que no consideran la diversidad ecológica en que crecen las diferentes especies y poblaciones de este género, ni permiten la experimentación o flexibilidad por parte de los dueños de los predios (Balvanera et al., 2009). El reto claramente aumenta cuando pasamos a otros ecosistemas boscosos. En los bosques tropicales, tanto húmedos como estacionalmente secos, el aprovechamiento forestal sigue siendo extractivo, aprovechando un número reducido de especies. Indudablemente, en estos ecosistemas es fundamental ampliar la visión de manejo para incorporar el aprovechamiento no extractivo (servicios ecosistémicos distintos al de provisión) y aumentar el número de especies potencialmente aprovechables (Rendón-Carmona, Martínez-Yrízar, Balvanera y Pérez-Salicrup, 2009).

Los recursos forestales, maderables y no maderables, de México ofrecen una amplia gama de opciones para diseñar el futuro del país. Así, de cerca de 7,000 especies de plantas que se aprovechan por las diferentes culturas de México, más del 90\% provienen de los bosques (Casas, Blancas y Lira, 2016). De este vasto mundo de recursos resulta prioritario identificar aquellos que se encuentran en mayor riesgo de extinción local o global, caracterizar las causas de este riesgo y actuar para impedir la desaparición y conservarlos. También resulta imperativo documentar las experiencias técnicas de manejo que ponen en práctica las comunidades de manejadores y contribuir a mejorar estas técnicas con base en investigación ecológica (tabla 3). Esta vertiente de investigación constituye una gran avenida de innovación ecotecnológica altamente prioritaria para asegurar el mantenimiento de los bosques y los múltiples servicios ecosistémicos que éstos proveen.

\section{Educación y evaluación de innovación ecotecnológica}

Con el fin de que las innovaciones ecotecnológicas para la sustentabilidad trasciendan con una verdadera apropiación social, se requiere de una estrategia y un acompañamiento fuerte de educación, capacitación, transferencia y monitoreo como se expone a continuación. 
Tabla 3

Reforestación comunitaria asistida dentro de la Reserva de la Biosfera Mariposa Monarca.

En México, los programas de reforestación rara vez tienen éxito debido a que son procesos unidireccionales donde solo se reporta el número de árboles plantados más no cuántos sobreviven. La Reserva de la Biosfera Mariposa Monarca (RBMM) en México se ha convertido en un buen ejemplo de esto. Cada año se plantan miles de árboles dentro de la Reserva y se paga a los miembros de las comunidades indígenas para que los planten. Sin embargo, no hay procesos de monitoreo de las tasas de supervivencia ni de crecimiento. Además, el suelo donde se plantan los árboles ya ha sufrido un proceso de erosión y degradación por lo que el éxito de las reforestaciones se reduce aún más. Se desarrolló un proyecto para restaurar un ecosistema forestal degradado a través de la recuperación de un suelo empobrecido mediante el uso de un abono orgánico preparado por miembros de una comunidad indígena que habita dentro de la RBMM (Jaramillo-López, Ramírez y Pérez-Salicrup, 2015). Se realizó la plantación de plántulas de Pinus pseudostrobus, que es la especie adecuada para la altitud y para la zona, en un suelo enmendado con el abono orgánico. Todo el proceso fue realizado por miembros de la comunidad indígena junto con científicos de la UNAM quienes tomaron en cuenta todas las variables necesarias para garantizar el éxito del proceso y se monitoreó durante 4 años. A lo largo del proceso, los miembros de la comunidad indígena se capacitaron para monitorear el crecimiento y la supervivencia de los árboles y con eso se logró un compromiso por parte de ellos logrando incrementar el éxito de la reforestación. La tasa de supervivencia luego de 3 años de establecido el experimento fue del $97 \%$. Esto hizo que los miembros de la comunidad indígena mantengan el interés ya que en pocos años podrían hacer el primer aprovechamiento de los árboles. Este modelo de compromiso comunitario y de éxito de la reforestación podría ser replicado en muchas zonas con similares características reduciendo la presión sobre los bosques protegidos y especialmente dentro de áreas naturales protegidas.

\section{Innovación educativa como parte de innovación ecotecnológica}

Hablar de innovación educativa implica proponer nuevas estrategias en el proceso de humanización, reconociendo a los humanos como seres cambiantes. Lo anterior es particularmente importante cuando se plantea la renovación de los procesos de enseñanza-aprendizaje que impulsan la innovación ecotecnológica para la sustentabilidad, pues no es suficiente la enseñanza de conocimientos y técnicas, sino también el desarrollo de habilidades críticas para la reflexión y la acción. Lo primero resultaría en una educación bancaria que domestica a quienes pretende «enseñar»; la segunda sería una educación humanista fundamentada en la investigación y la práctica política (tabla 4).

La posibilidad real de innovar, educar y capacitar, así como lograr innovación ecotecnológica y consolidarla a mediano y largo plazo, requiere de 2 procesos fundamentales: $i$ ) involucrar a la gente en esquemas colaborativos de investigación-acción (Salazar, 1992) y ii) desarrollar programas educativos adecuados a las condiciones socioculturales de las poblaciones con las que se colabora, en los que se reconozcan plenamente a los interlocutores como sujetos y que promuevan su empoderamiento (Freire, 1970). Para ello se requiere de abordajes transdisciplinarios, los cuales parten del paradigma de la complejidad (Morin, 1995). Desde lo pedagógico, el marco que se propone para el desarrollo de dichos procesos es el del constructivismo. En este, se desarrollan procesos horizontales y se promueve un
Tabla 4

La meliponicultura: patrimonio biocultural y herramienta pedagógica.

La innovación ecotecnológica se facilita extraordinariamente si se trabaja a partir de Investigación Acción Participativa (IAP) acompañada de procesos educativos. Por ejemplo, desde el 2014 en Tabasco se ha desarrollado una propuesta de IAP con el objetivo principal de promover prácticas agroecológicas a partir del desarrollo de ciclos de reflexión-acción-reflexión con un enfoque basado en el concepto de paisaje cultural (Schaich, Bieling y Plieninger, 2010), el paradigma de la complejidad (Morin, 1995) y el análisis del cultivo de abejas nativas sin aguijón (meliponicultura). Las abejas nativas tienen una gran importancia ecológica como polinizadores, lo que permite el fácil abordaje de temas de agrodiversidad e impactos del uso de agroquímicos en eventos de intercambio de saberes. Asimismo, el cultivo de abejas representa un recurso importante para las familias rurales ya que a la miel se le dan diferentes 881 usos medicinales. A mediano plazo puede representar también un recurso económico ya que diversos productos meliponinos son muy apreciados: además de la miel, el polen, la cera y el propóleo. En el proyecto se ha trabajado en la construcción de conocimiento y se han detonado procesos de reflexión en torno a la situación socioambiental, a partir del diálogo de saberes (Rosset y Martínez-Torres, 2013), aplicadas a la meliponicultura. Las metodologías utilizadas están basadas en las ideas de Freire (1970) y retoman los 4 pilares de la educación propuestos por Delors (1996). Partiendo de la documentación de los conocimientos, prácticas y creencias (Berkes, 1999; Aldasoro, 2012), en comunidades tabasqueñas en montañas bajas y planicie costera, el aprendizaje colectivo sobre la meliponicultura se convierte en una herramienta pedagógica eficiente para propiciar la conservación de especies melíferas en el paisaje productivo, valorar el conocimiento agroecológico local y el diseño de alternativas ecotecnológicas

aprendizaje significativo. En este aprendizaje los participantes construyen su conocimiento a partir del establecimiento de relaciones entre los conceptos a aprender y los que ya se tienen (Carretero, 2009). Los individuos aprenden a conocer, a ser, a hacer y a convivir con los otros, cultivando ideales de paz, de libertad y de justicia social (Delors, 1996). El constructivismo facilita el establecimiento de diálogos de saberes (Freire, 1970; Rosset y Martínez-Torres, 2013), a partir de los cuales se retoman y revaloran los saberes campesinos/indígenas contemporáneos al mismo tiempo que se establece el intercambio con los conocimientos científicos. Los procesos de enseñanzaaprendizaje implican entonces no solo aspectos cognitivos, sino también emocionales e incluso espirituales. A partir de ello, es posible promover cambios sociales que aporten elementos para la superación de crisis ambientales y socioecológicas y, al mismo tiempo, fortalecer la recuperación de la sabiduría colectiva y la inteligencia social (Del Amo-Rodríguez, 2012).

La innovación en educación debe desarrollarse en el enfoque, en el método, en los educadores, en los receptores de la educación y en las herramientas didácticas. La educación no es solo para los receptores de las innovaciones ecotecnológicas (los niños, los estudiantes y los productores). La comunidad científica rara vez se identifica como un recipiente lógico de la educación, porque ya se considera ampliamente educada; sin embargo, hay conocimiento cultural, emocional y tradicional (particularmente de interacciones socioecológicas) que desconoce, las cuales tienen mucho peso en las decisiones y valores de quienes manejan los recursos naturales, los ecosistemas 
naturales, los sistemas productivos, las empresas, etc. También, con mucha frecuencia, tiene desconocimiento del trabajo multi-, inter- y transdisciplinario, del pensamiento complejo, de formas alternativas de hacer investigación, de vincularse con la sociedad y de formar recursos humanos con estas propiedades. En la innovación ecotecnológica, la educación y la capacitación debe ser continua y para todos los actores involucrados.

Es necesario transformar los programas de entrenamiento tradicional e incorporar habilidades novedosas para la resolución de problemas y nuevas perspectivas socioecológicas para fortalecer exitosamente la capacitación para la innovación ecotecnológica y la sustentabilidad (Clark, van Kerkhoff, Lebel y Gallopin, 2016). Hay 3 competencias críticas para fortalecer el entrenamiento práctico: $i$ ) pensamiento en sistemas complejos, ii) colaboración entre múltiples actores y herramientas participativas y iii) perspectiva interdisciplinaria (Wiek, Withycombe y Redman, 2011).

\section{Monitoreo y sistematización de conocimientos y experiencias}

El proceso de implementación de innovaciones ecotecnológicas requiere trascender el concepto convencional de transferencia tecnológica para asegurar que los impactos que se esperan se materialicen efectivamente al ser aplicadas en la comunidad. Los beneficios esperados ocurren solo si las ecotecnologías funcionan y mantienen su desempeño técnico para resolver el problema deseado, y si las comunidades que las usan se apropian de ellas, las hacen parte de sus vidas, las evalúan, las mantienen, las mejoran o las generan (Ruiz-Mercado y Masera, 2015). La forma en que ocurren los procesos ecotecnológicos de innovación, adopción y apropiación determina de manera crítica la funcionalidad que, en el largo plazo, tendrán las ecotecnologías para cualquier beneficio ambiental o socio-ecológico tangible (Ruiz-Mercado, Masera, Zamora y Smith, 2011).

En el contexto anterior, el monitoreo participativo, bajo un modelo de laboratorio abierto, es fundamental para mejorar las implementaciones y asegurar que en realidad las ecotecnologías robustecen los sistemas socioecológicos, o más bien sociotecnoecológicos (SETS). El monitoreo ecotecnológico tiene como finalidad primaria la caracterización de los procesos -como la innovación, la adopción, la apropiación- que tienen lugar en los SETS, así como la medición de sus parámetros clave y la formulación de acciones, rutas críticas y protocolos para asegurar la función de las ecotecnologías como herramientas para la sustentabilidad de los sistemas socioecológicos en los que son implementadas (Ruiz-Mercado et al., 2011). El monitoreo favorece la capacidad de adaptación de los SETS y genera evidencia firme de casos exitosos para generar políticas públicas o consolidar financiamientos, además de que puede por sí mismo ser un catalizador de otros procesos de cohesión y participación comunitaria. El monitoreo es por ello un elemento esencial en la innovación ecotecnológica. Para asegurarla, se requiere fortalecer la formación de recursos humanos en este campo, acelerar las colaboraciones inter- y transdisciplinarias para generar marcos teóricos y nuevas formas de trabajo y el desarrollo de herramientas y metodologías para la recolección, análisis, síntesis y utilización de datos enfocados al diagnóstico oportuno, monitoreo costo-efectivo y de largo plazo, que permite evaluar, corregir, adaptar, para mejorar continuamente las innovaciones (Wigboldus et al., 2016).

\section{Reflexión general y conclusiones}

Las herramientas y las innovaciones ecotecnológicas son motores de transformación ambiental y socioecológica y deben crearse por la conjunción de las capacidades científicas y técnicas. La comunidad científica (entre ellos los ecólogos) es indispensable en este proceso, pero hasta ahora se ha mantenido distante y desconectada del proceso de crear alternativas para la sustentabilidad. Esto se debe, en parte, a la falta de incentivos para su involucramiento con otros actores de la sociedad, ya que la publicación científica prevalece como el criterio principal de desarrollo académico. Por ello, no sorprende que las publicaciones sean el único producto de proyectos de investigación que supuestamente se financiaron para brindar soluciones a sectores productivos como el agrícola, el pecuario, la acuícola y el agrobiotecnológico (Amaro-Rosales y Villavicencio-Carbajal, 2015). Más aún, los biólogos y los ecólogos, al contrario de los ingenieros, físicos o químicos, a menudo consideraban los desarrollos tecnológicos como productos de «segunda clase» y cuestionaban sus méritos hasta que se volvieron un cuadro para rellenar en las evaluaciones del Sistema Nacional de Investigadores (un programa de becas de desempeño del Consejo Nacional de Ciencia y Tecnología de México). Gradualmente, las disciplinas menos tecnológicas, como aquellas de las ciencias naturales, empiezan a reconocer que la innovación y la tecnología son mucho más que patentes y máquinas. En un mundo que enfrenta cada vez más problemas socioecológicos y ambientales, se está enfatizando la importancia de que la comunidad científica sea creativa e innovadora de métodos, procesos y herramientas para hacer mejor ciencia básica y aplicada con mayores contribuciones a la sociedad. Obviamente, esto debe implicar también la plena participación de instituciones públicas, así como el establecimiento de políticas de cambios y de apoyo a estos nuevos modelos de investigación.

Los científicos a nivel mundial deberían involucrarse más en la construcción de la sustentabilidad. Los ecólogos mexicanos deberíamos comprometernos más en innovar nuestras maneras de hacer investigación y nuestras metas como científicos y contribuir al desarrollo sustentable, mejorando o desarrollando alternativas a los métodos, enfoques y estándares internacionales que no corresponden a nuestro contexto socioecológico. Probablemente, el mayor reto que tienen los científicos en el futuro cercano, para contribuir a la construcción de la sustentabilidad, es el de trabajar juntos y al mismo nivel con los políticos, los productores, los empresarios, los maestros, las comunidades rurales y urbanas, entre otros actores y sectores sociales.

\section{Agradecimientos}

Este artículo es resultado del trabajo de algunos participantes del Laboratorio Nacional de Innovación Ecotecnológica para la Sustentabilidad (LANIES) con financiamiento del Consejo Nacional de Ciencia y Tecnología, México. Los autores 
agradecen el financiamiento del proyecto Conacyt-LABNAL2016-271570 y del programa de Cátedras Conacyt otorgadas a: E.M.A., B.A.O., M.C.P., P.J.L., F.P., I.R.M.

\section{Referencias}

Aldasoro, M. E. M. (2012). Documenting and contextualizing Pjiekakjoo (Tlahuica) knowledges through a Research Project (Tesis doctoral). Seattle, Washington, EUA: University of Washington.

Altieri, M., Nicholls, C., Henao, A. y Lana, M. (2015). Agroecology and the design of climate change resilient farming systems. Agronomy for Sustainable Development, 35, 869-890.

Amaro-Rosales, M. y Morales-Sánchez, M.A. (2010). La biotecnología en México, una aproximación desde los sistemas sectoriales de innovación. Ide@s CONCYTEG, 5.

Amaro-Rosales, M. y Villavicencio-Carbajal, D. H. (2015). Incentivos a la innovación de la biotecnología agrícola alimentaria en México. Estudios Sociales, 23, 34-62.

Amézquita, M. C., Ibrahim, M., Llanderal, T., Buurman, P. y Amézquita, E. (2004). Carbon sequestration in pastures, silvo-pastoral systems and forests in four regions of the Latin American Tropics. Journal of Sustainable Forestry, 21, 31-49.

Balvanera, P., Cotler, H., Aburto, O., Aguilar, A., Aguilera, M., Aluja, M., et al. (2009). Estado y tendencias de los Servicios Ecosistémicos. En R. Dirzo, R. González, y I. March (Eds.), Capital Natural y Bienestar Social: segundo estudio de país. (pp. 185-245). Segunda Parte. México D.F.: Comisión Nacional para el Conocimiento y Uso de la Biodiversidad.

Berkes, F. (1999). Sacred ecology: traditional ecological knowledge and management systems. Filadelfia, Londres: Taylor and Francis.

Bunge, V., Cotler, H., Iura-González, D. y Ruiz-Bedolla, K. (2014). Las cuencas en los instrumentos de planeación ambiental: nuevos retos. En F. PérezCorrea (Ed.), Gestión pública y social del agua en México (pp. 12-20). México D.F.: Universidad Nacional Autónoma de México.

Cannatelli, B., Masi, A. G. y Molteni, M. (2012). Green technology implementation in developing countries: opportunity identification and business model design. En A. Nicholls y A. Murdoch (Eds.), Social innovation (pp. 248-269). Londres: Palgrave Macmillan.

Carpenter, S. H. y Gunderson, L. H. (2001). Coping with collapse: ecological and social dynamics in ecosystem management. Bioscience, 51, 451-457.

Carretero, M. (2009). Constructivismo y educación. Buenos Aires: Paidós.

Casas, A., Blancas, J. y Lira, R. (2016). Mexican ethnobotany. Interactions of people and plants in Mesoamerica. En R. Lira, A. Casas, y J. Blancas (Eds.), Ethnobotany of Mexico. Interactions of peoples and plants in Mesoamerica (pp. 1-19). Utrecht, Holanda: Springer.

Challenger, A. y Dirzo, R. (2009). . Factores de cambio y estado de la biodiversidad Capital Natural de México, Vol II: Estado de conservación y tendencias de cambio (II) México D.F.: Comisión Nacional para el Conocimiento, Uso y Manejo de la Biodiversidad.

Cho, D., Ogwang, T. y Opio, C. (2010). Simplifying the water poverty index. Social Indicators Research, 97, 257-267.

Clark, W. C., van Kerkhoff, L., Lebel, L. y Gallopin, G. C. (2016). Crafting usable knowledge for sustainable development. Proceedings of the National Academy of Sciences, 113, 4570-4578.

Conabio. (1998). La diversidad biológica de México: estudio de país. México D.F.: Comisión Nacional para el Conocimiento y Uso de la Biodiversidad.

Cotler, H., Sotelo, E., Domínguez, J., Zorrilla, M., Cortina, S. y Quiñones, L. (2007). La conservación del suelo: un asunto de interés público. Gaceta Ecológica, 83, 5-71.

Crutzen, P. J. y Stoermer, E. F. (2000). The'Anthropocene'. Global Change Newsletter, 41, 17-18.

Del Amo-Rodríguez, S. (2012). El repoblamiento del área rural, recuperando la sabiduría colectiva y la inteligencia social. Estrategias participativas de Investigación-acción para la intervención local. México D.F.: Plaza y Valdez.

Delgado-Lemus, A., Torres, I., Blancas, J. y Casas, A. (2014). Vulnerability and risk. Journal of Ethnobiology and Ethnomedicine, 10, 53 .
Delors, J. (1996). La educación encierra un tesoro. México D.F.: Ediciones UNESCO.

Dirzo, R., González, R., y March, I. (Eds.). (2009). Capital natural y bienestar social: segundo estudio de país. México D.F.: Comisión Nacional para el Conocimiento y Uso de la Biodiversidad.

Espinoza-Tenorio, A., Mesa-Jurado, M. A., Ortega-Argueta, A. y HernándezChávez, M. (2017). Laboratorios para la sustentabilidad: nuevos espacios para el quehacer científico y la formación de recursos humanos. Revista de El Colegio de San Luis, 13, 1-23.

Evenson, R. E. y Gollin, D. (2003). Assessing the impact of the Green Revolution, 1960 to 2000. Science, 300, 758-762.

Ferguson, B. G., Diemont, S., Alfaro-Argüelles, R., Martinc, J., Nahed-Toral, J., Álvarez-Solís, D., et al. (2013). Sustainability of holistic and conventional cattle ranching in the seasonally dry tropics of Chiapas, Mexico. Agricultural Systems, 120, 38-48.

Freire, P. (1970). Pedagogía del oprimido. Madrid: Siglo XXI.

Fressoli, M., Dias, R. y Thomas, H. (2014). Innovation and Inclusive development in the south: a critical perspective. En E. Medina, I. da Costa-Marques, y C. Holmes (Eds.), Beyond imported magic. Essays on science, technology, and society in Latin America (pp. 45-63). Cambridge, MA: MIT Press.

Fressoli, M., Smith, A., Thomas, H. y Bortz, G. (2015). De las tecnologías apropiadas a las tecnologías sociales: algunos dilemas persistentes de los movimientos alternativos de innovación. En H. Thomas, F. Picabea, y B. Albornoz (Eds.), Aproximaciones al CTS en América Latina. Quito: FLACSO Ecuador.

García, R. y Calantone, R. (2002). A critical look at technological innovation typology and innovativeness terminology: a literature review. Journal of Product Innovation Management, 19, 110-132.

Gupta, A. K., Sinha, R., Koradia, D., Patel, R., Parmar, M., Rohit, P., et al. (2003). Mobilizing grassroots' technological innovations and traditional knowledge, values and institutions: articulating social and ethical capital. Futures, 35, 975-979.

Huber-Sannwald, E., Maestre, F. T., Herrick, J. E. y Reynolds, J. F. (2006). Ecohydrological feedbacks and linkages associated with land degradation: a case study from Mexico. Hydrological processes, 20, 3395-3411.

Ibrahim, M., Villanueva, C., Casasola, F. y Rojas, J. (2006). Sistemas silvopastoriles como una herramienta para el mejoramiento de la productividad y restauración de la integridad ecológica de paisajes ganaderos. Pastos y Forrajes, 29, 383-419.

Jaramillo-López, P. F., Ramírez, M. I. y Pérez-Salicrup, D. R. (2015). Impacts of Bokashi on survival and growth rates of Pinus pseudostrobus in community reforestation projects. Journal of Environmental Management, 150, 48-56.

Jiménez-Ferrer, G., Aguilar-Argüello, V. y Soto-Pinto, L. (2008). Livestock and carbon sequestration in the Lacandon rainforest, Chiapas, Mexico. En P. Rowlinson, M. Steele, y A. Nefzaoui (Eds.), Proceedings of the Internacional Conference Livestock and Global Climate Change (pp. 195-197). Hammamet, Tunisia: Cambridge University Press.

Lambin, E. F., Turner, B. L., Geist, H. J., Agbola, S. B., Angelsen, A., Bruce, J. W., et al. (2001). The causes of land-use and land-cover change: moving beyond the myths. Global Environmental Change, 11, 261-269.

Levins, R. y Lewontin, R. C. (1985). The dialectical biologist. Harvard: Harvard University Press.

Mahecha, L. (2016). El silvopastoreo: una alternativa de producción que disminuye el impacto ambiental de la ganadería bovina. Revista Colombiana de Ciencias Pecuarias, 15, 226-231.

Martínez-Torres, H. L., Cantú-Férnandez, M. X., Ramírez-Ramírez, M. I. y Pérez-Salicrup, D. R. (2015). Fires and fire management in the Monarch Butterfly Biosphere Reserve. En K. Oberhauser, K. R. Nail, y S. Altizer (Eds.), Monarchs in a changing world: biology and conservation of an iconic butterfly (pp. 179-189). Ithaca: Cornell University Press.

Martínez-Torres, H. L., Castillo, A., Ramírez-Ramírez, M. I. y Pérez-Salicrup, D. R. (2016). Traditional fire knowledge system in a subtropical montane socioecosystem in a natural protected area. International Journal of Wildland Fire, 25, 911-925.

Millennium Ecosystem Assessment (MEA). (2005). Ecosystems and human well-being: synthesis. Washington, D.C: Island Press.

Morin, E. (1995). Introducción al pensamiento complejo. España: Gedisa. 
Murgueitio, E. y Ibrahim, M. (2001). Agroforestería pecuaria para la reconversión de la ganadería en Latinoamérica. Livestock Research for Rural Development, 13, 1-12.

Nahed-Toral, J., Sánchez-Muñoz, B., Mena, Y., Ruiz-Rojas, J., Aguilar-Jiménez, R., Castel, M. J., et al. (2013). Feasibility of converting agrosilvopastoral systems of dairy cattle to the organic production model in southeastern Mexico. Journal of Cleaner Production, 43, e145.

Olsson, P. y Galaz, V. (2012). Social-ecological innovation and transformation. En A. Nicholls y A. Murdoch (Eds.), Social innovation (pp. 223-247). Londres: Palgrave Macmillan.

ONU (Organización de las Naciones Unidas). (2015). Transforming our world: the 2030 Agenda for sustainable development. A/RES/70/1. United Nations [consultado 21 Oct 2016]. Disponible en: www.sustainabledevelopment.un.org.

Opio, C., Gerber, P., Mottet, A., Falcucci, A., Tempio, G., MacLeod, M., et al. (2013). Greenhouse gas emissions from ruminant supply chains - a global life cycle assessment. Roma: Food and Agriculture Organization of the United Nations (FAO).

Ortiz, J., Masera, O. y Fuentes, A. (2014). La ecotecnología en México. México D.F.: Imagia Comunicación.

Ostrom, E. (2007). A diagnostic approach for going beyond panaceas. Proceedings of the National Academy of Sciences, 104, 15181-15187.

Ostrom, E. (2009). A general framework for analyzing sustainability of socialecological systems. Science, 325, 419-422.

Pérez-Salicrup, D. R. (2005). La restauración en relación con el uso extractivo de recursos bióticos. En O. Sánchez, E. Peters, R. Márquez-Huitzil, E. Vega, G. Portales, y M. Valdez, et al. (Eds.), Temas sobre restauración ecológica (pp. 79-86). México D.F.: Instituto Nacional de Ecología, Semarnat, U.S. Fish \& Wildlife Service, Unidos para la Conservación, A.C.

Perfecto, I. y Vandermeer, J. (2015). Coffee agroecology. A new approach to understanding agricultural biodiversity, ecosystem services and sustainable development. Londres: Routledge, Earthscan.

Perfecto, I., Vandermeer, J. y Wright, A. (2009). Nature's matrix: linking agriculture conservation and food sovereignty. Londres: Routeledge, Earthscan.

Pimentel, D., Harvey, C., Resosudarmo, P., Sinclair, K., Kurz, D., McNair, M., et al. (1995). Environmental and economic costs of soil erosion and conservation benefits. Science, 267, 1117-1123.

Pinard, M. A., Guariguata, M. R., Putz, F. E. y Pérez-Salicrup, D. R. (2006). Managing natural tropical forests for timber: experiences, challenges and opportunities. En M. J. Groom, G. K. Meffe, y C. R. Carroll (Eds.), Principles of conservation biology (pp. 283-290). Sunderland: Sinauer Associates, Inc.

Pingali, P. L. (2012). Green revolution: impacts, limits, and the path ahead. Proceedings of the National Academy of Sciences, 109, 12302-12308.

Rangel-Landa, S., Dávila, P. y Casas, A. (2015). Facilitation of A gave potatorum: an ecological approach for assisted population recovery. Forest Ecology and Management, 347, 57-74.

Redman, C. L. y Miller, T. R. (2015). The technosphere and Earth Stewardship. En R. Rozzi, F. S. Chapin III, J. B. Callicot, S. T. A. Picket, M. E. Power, y J. J. Arnesto, et al. (Eds.), Earth Stewardship, ecology and ethics (2) (pp. 269-279). Dordrecht, Suiza: Springer International Publishing.

Rendón-Carmona, H., Martínez-Yrízar, A., Balvanera, P. y Pérez-Salicrup, D. R. (2009). Selective cutting of woody species in a Mexican tropical dry forest: incompatibility between use and conservation. Forest Ecology and Management, 257, 567-579.

Rosset, P. M. y Martínez-Torres, M. E. (2013). Rural social movements and diálogo de saberes: territories, food sovereignty and agroecology. In En Food sovereignty: a critical dialogue International Conference. Yale: Yale University.

Ruiz-Mercado, I. y Masera, O. (2015). Patterns of stove use in the context of fuel-device stacking: rationale and implications. EcoHealth, 12, 42-56.
Ruiz-Mercado, I., Masera, O., Zamora, H. y Smith, K. R. (2011). Adoption and sustained use of improved cookstoves. Energy Policy, 39, 7557-7566.

Salazar, M. C. (1992). La investigación-acción participativa. Inicios y desarrollos. Madrid: Editorial Popular.

Schaich, H., Bieling, C. y Plieninger, T. (2010). Linking ecosystem services with cultural landscape research. Gaia-Ecological Perspectives for Science and Society, 19, 269-277.

Semarnat (Secretaría de Medio Ambiente y Recursos Naturales). (2014a). Programa Nacional Hídrico 2014-2018. México, D.F. [consultado 13 Oct 2016]. Disponible en: http://www.conagua.gob.mx/CONAGUA07/ Contenido/Documentos/PROGRAMA_Nacional_Hidrico_2014_2018_espa $\% \mathrm{C} 3 \% \mathrm{~B} 1 \mathrm{ol} . \mathrm{pdf}$

Semarnat (Secretaría de Medio Ambiente y Recursos Naturales). (2014 b). Anuario estadístico de la producción forestal 2013. México, D.F.: Dirección General de Gestión Forestal y de Suelos, Dirección del Registro y del Sistema Nacional de Gestion Forestal.

Smith, A. y Stirling, A. (2010). The politics of social-ecological resilience and sustainable socio-technical transitions. Ecology and Society, 15, 11.

Steinfeld, H. y Chilonda, P. (2006). Old players new players. En A. McLeod (Ed.), The 2006 livestock report (pp. 3-14). Rome: Food and Agriculture Organization.

Straškava, M. (1993). Ecotechnology as a new means for environmental management. Ecological Engineering, 2, 311-331.

Tapia-Tapia, E. C. y Reyes-Chipla, R. (2008). Productos forestales no maderables en México: aspectos económicos para el desarrollo sustentable. Madera y Bosques, 14, 95-112.

Thomas, H. (2012). Tecnologías para la inclusión social en América Latina: de las tecnologías apropiadas a los sistemas tecnológicos sociales. Problemas conceptuales y soluciones estratégicas. En H. (Org. ) Thomas, G. Santos, y M. Fressoli (Eds.), Tecnología, desarrollo y democracia. Nueve estudios sobre dinámicas socio-técnicas de exclusión/inclusión social (pp. 25-78). Buenos Aires: MINCyT.

Torres, I., Blancas, J., León, A. y Casas, A. (2015). TEK, local perceptions of risk, and diversity of management practices of Agave inaequidens in Michoacán, Mexico. Journal of Ethnobiology and Ethnomedicine, 11, 61.

Torres, I., Casas, A., Delgado-Lemus, A. y Rangel-Landa, S. (2013). Aprovechamiento, demografía y establecimiento de Agave potatorum en el valle de Tehuacán, México: aportes etnobiológicos y ecológicos para su manejo sustentable. Zonas Áridas, 15, 92-109.

Torres, I., Casas, A., Vega, E., Martínez-Ramos, M. y Delgado-Lemus, A. (2015). Population dynamics and sustainable management of mescal agaves in central Mexico: Agave potatorum in the Tehuacán-Cuicatlán Valley. Economic Botany, 69, 26-41.

Tudela, F. (1992). La modernización forzada del trópico: el caso tabasqueño México D.F.: Proyecto Integrado del Golfo, El Colegio de México, CINVESTAV, IFIAS, UNRISD.

Vega, M. A. (2012). Aspectos y avances en ciencia, tecnología e innovación. Polis (Santiago), 11, 451-470.

Villanueva, L. G., Martínez, Z. P., Casanova, L. F., Ramírez, A. L. y Montañez, E. P. I. (2015). Carbon storage in livestock systems with and without live fences of Gliricidia sepium in the humid tropics of Mexico. Agroforestry Systems, 89, 1083-1096.

Wiek, A., Withycombe, L. y Redman, C. L. (2011). Key competencies in sustainability: a reference framework for academic program development. Sustainability Science, 6, 203-218.

Wigboldus, S., Klerkx, L., Leewis, C., Schut, M., Muilerman, S. y Jochemsen, H. (2016). Systemic perspectives on scaling agricultural innovations. A review. Agronomy for Sustainable Development, 46, 1-20.

Zika, M. y Erb, K. (2009). The global loss of net primary production resulting from human-induced soil degradation in drylands. Ecological Economics, $69,310-318$ 